\title{
DESCRIPCIÓN DE LOS INSTRUMENTOS DE MEDIDA DE LA MOVILIDAD EN PERSONAS MAYORES DE 65 AÑOS. REVISIÓN SISTEMÁTICA
}

\author{
Francisco Javier Rubio Castañeda (1), Concepción Tomás Aznar (1), Carmen Muro Baquero (1) y \\ Johanna Chico Guerra (2).
}

(1) Departamento de Enfermería y Fisiatría. Universidad de Zaragoza

(2) Hospital Clínico Universitario Lozano Blesa de Zaragoza

\section{RESUMEN}

Fundamentos: Las personas mayores de 65 años son quienes presentan diferentes niveles de limitaciones funcionales. Existen múltiples instrumentos de medición de la movilidad en este grupo de edad y una falta de consenso internacional sobre cuáles son los más idóneos. El objetivo fue describir los instrumentos de medición de la movilidad en personas mayores de 65 años, determinar sus ventajas y limitaciones y comparar las características de validez de cada instrumento para poder establecer cuáles son los más válidos para este fin.

Métodos: Revisión sistemática de la bibliografía sobre instrumentos utilizados para medir la movilidad en personas mayores de 65 años publicados entre 2001 a 2013. La búsqueda se realizó en las bases de datos Pubmed y Science Direct.

Resultados: Los 34 artículos seleccionados permitieron agrupar los instrumentos en 4 grupos: agua doblemente marcada (DLW), detectores de movimiento, medidas objetivas de la movilidad y cuestionarios. Del total de artículos, 23 correspondieron a cuestionarios, 4 fueron sobre acelerómetros, 3 sobre medidas objetivas de la movilidad y 2 tanto sobre podómetros como DLW. Los otros 2 hacían referencia al análisis combinado de diferentes instrumentos.

Conclusiones: Las medidas de movilidad más precisas se obtienen evaluando datos objetivos y subjetivos. Para obtener datos objetivos se usan las medidas objetivas de la movilidad frente a podómetros y acelerómetros, mientras que los cuestionarios son utilizados para obtener datos subjetivos debido a su facilidad de uso y a los datos sociodemográficos que aportan. Entre los instrumentos, el Short Physical Performance Battery (SPPB) y el Minesotta Leisure Time resultan los instrumentos más idóneos para medir la movilidad de las personas mayores en España.

Palabras clave: Actividad física. Actividad motora. Cuestionarios. Ancianos. Validez. Fiabilidad.

Correspondencia

Francisco Javier Rubio Castañeda

Dirección: Avenida Cardenal Cisneros $\mathrm{N}^{\circ} 18,1^{\circ} \mathrm{C}$

34004. Palencia

Correo electrónico: fjrubio.due@gmail.com

DOI:

\section{ABSTRACT \\ Mobility Assessment in Elderly People. Description OF Measuring Instruments for Mobility. A Review}

Background: People over 65 are the ones who have higher levels of functional limitations. There are many instruments to measure mobility in this age range that causes a lack of international consensus on what are the most suitable for this purpose. The aim is to analyze and identify which instruments provide greater data reliability and validity in measuring mobility in elderly people.

Methods: Systematic review of the instruments used to measure mobility in people over 65 years published between 2001-2013, conducted in PubMed and Science Direct.

Results: The 34 items selected instruments were grouped into 4 categories: doubly labeled water (DLW), motion detectors, objective measures of mobility and questionnaires. We identified, 23 are questionnaires, 4 accelerometers, 3 to objective measures of mobility and 2 both pedometers as DLW. The other 2 to combined analysis of different instruments.

Conclusions: We conclude that assessing objective and subjective data obtains the most accurate measures of mobility. To obtain objective data, objective measures of mobility will be opposed to pedometers and accelerometers, while questionnaires were selected for subjective data due to its ease of use and sociodemographic data that provides. Among these instruments, the Short Physical Performance Battery (SPPB) and Minnesota Leisure Time are the most appropriate instruments to estimate the mobility of the elderly in Spain.

Keywords: Physical activity. Motor Activity. Questionnaire . Elderly. Validity. Reliability. 


\section{INTRODUCCIÓN}

La movilidad es la habilidad para moverse, desplazarse o valerse por uno mismo dentro del hogar, el vecindario o el entorno y permite la independencia individual ${ }^{1}$. Para una correcta movilidad es imprescindible el adecuado funcionamiento de las extremidades superiores e inferiores. Cuando existen problemas de funcionalidad en cualquiera de las extremidades, la capacidad de las personas para moverse dentro de su entorno de forma autónoma e independiente queda limitada, dando lugar al desarrollo de limitaciones funcionales ${ }^{2,3}$. Las limitaciones de la movilidad son uno de los primeros estadios del proceso de discapacidad, de hecho constituyen el componente principal de los modelos que explican la génesis de la discapacidad. En dichos modelos se considera que los problemas de la movilidad son el estado de transición entre las etapas de deficiencia y de discapacidad ${ }^{4-6}$. Cuando una persona sufre limitaciones funcionales su calidad de vida, salud física y mental y la realización de las actividades básicas e instrumentales de la vida diaria se verán afectadas, incrementándose el riesgo de discapacidades, incapacidades, institucionalización y de muerte ${ }^{7-9}$.

La incidencia y prevalencia de limitaciones funcionales está influida por la edad y por factores de tipo cognitivo, psicosocial, ambiental, físicos, financieros, de género, culturales e individuales ${ }^{4}$. En función de la edad, son las personas $\geq 65$ años quienes presentan un mayor nivel de limitaciones funcionales $^{10,11}$, de hecho entre un tercio y la mitad de las personas mayores de 65 años perciben una pérdida de movilidad en actividades cotidianas como caminar o subir escaleras ${ }^{10}$. La mayor pérdida de movilidad se debe principalmente al proceso natural de envejecimiento que provoca pérdida de masa muscular y densidad ósea así como erosiones articulares, limitando todo ello la movilidad tanto en el hogar como en la comunidad $^{10,12}$. Además de la afectación fisio- lógica que la edad produce en la movilidad, los factores socioculturales y las condiciones crónicas agravan la pérdida de movilidad de las personas mayores al afectar más severamente a su movilidad, esto se debe a que son más vulnerables a los factores del medio que los rodea ${ }^{2}$.

Para detectar la pérdida de movilidad y poder prevenirla existe un elevado número de instrumentos de medida de diferente naturaleza, agua doblemente marcada (DLW), detectores de movimiento (podómetros y acelerómetros), medidas objetivas de la movilidad (funcionales) y cuestionarios. Aspectos tan diversos como el lugar de colocación (sensores de movimiento) o el tipo de variables que miden influyen en los datos de movilidad obtenidos y dificultan la obtención de los resultados válidos. Ello genera una falta de consenso internacional sobre cuál de estos instrumentos es más preciso y fiable ${ }^{13}$.

También existen discrepancias internacionales sobre la definición de movilidad. Diversos cuestionarios determinan la medida de la movilidad desde distintas perspectivas y ámbitos: gasto energético (agua doblemente marcada, acelerómetros y cuestionarios), actividad física (podómetros y cuestionarios) y medidas de movilidad funcional tanto objetivas como subjetivas. Por ejemplo, caminar, que es una de las principales formas de movilidad entre las personas mayores, informa tanto sobre movilidad como sobre actividad física $(\mathrm{AF})^{1}$, siendo empleada tanto por cuestionarios que miden AF como movilidad. La relación entre movilidad, AF y gasto energético se puede observar en la figura 1.

El objetivo de este estudio fue describir los instrumentos de medición de la movilidad en personas mayores de 65 años, determinar sus ventajas y limitaciones y comparar las características de validez de cada instrumento para poder establecer cuáles son los más válidos para este fin. 


\section{Figura 1}

\section{Relación entre actividad física, movilidad y gasto energético}

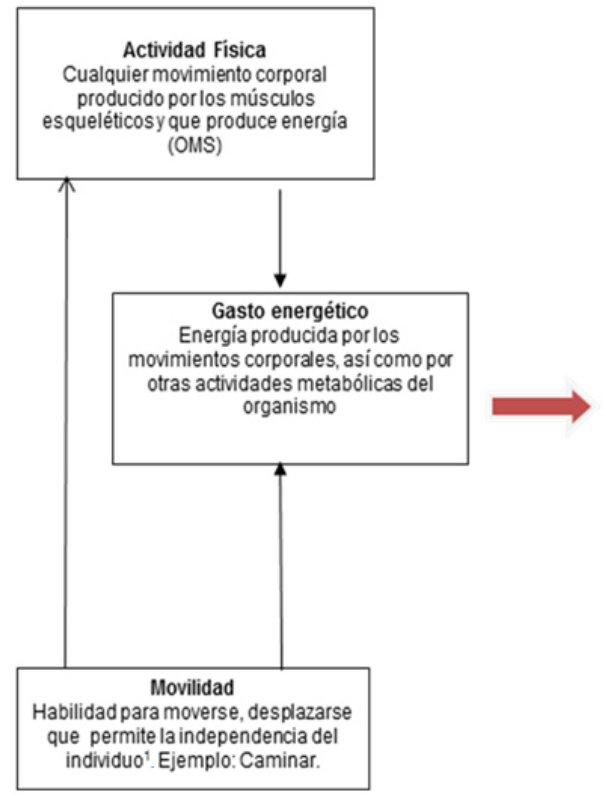

\section{MATERIAL Y MÉTODOS}

Diseño. Revisión sistemática de los artículos sobre instrumentos utilizados para medir la movilidad en personas mayores de 65 años. Se realizó un análisis de su validación así como de las características de cada uno de los instrumentos estudiados.

Estrategia de búsqueda. La búsqueda bibliográfica se realizó en las bases de datos Pubmed y Science Direct. La primera fase comenzó usando las palabras clave "mobility, movement, functional status, physical activity, physical function" para evaluar ampliamente todo el rango de movilidad y se cruzó con los resultados obtenidos con las palabras clave que hicieron referencia a la evaluación o análisis de la movilidad: "mobility monitoring, mobility measures, scales, geriatric assessment y assessment". A cada uno de estos grupos de palabras se les definió como B1 y B2 respectivamente. Tras esta búsqueda se obtuvieron las

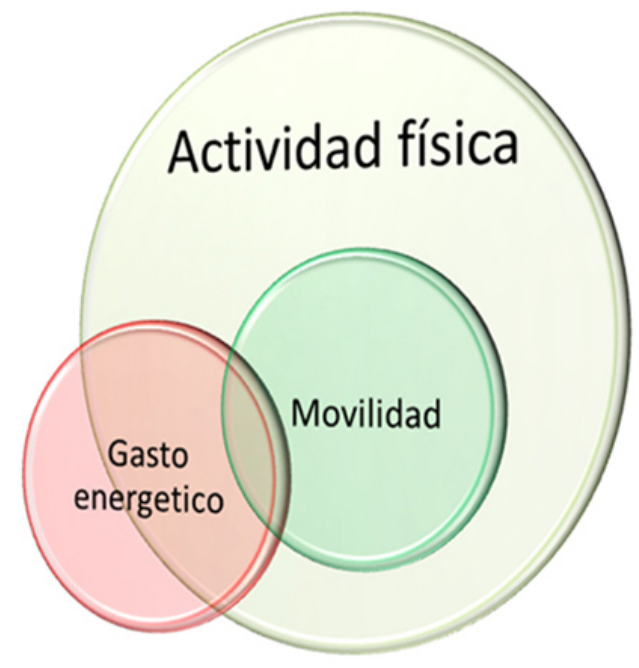

palabras claves que permitieron localizar los artículos sobre los instrumentos que se analizaron en este estudio: "questionnaire, self report measures, accelerometer, accelerometry, pedometer, objective measures, doble-labeled water (DLW). Dichas palabras que definimos como B3 fueron cruzadas con las de la población diana de este estudio: "geriatric, older people y elderly" que definimos como B4. Para cada una de estas búsquedas se empleó el operador OR entre todas las palabras clave de B1, B2, B3 y B4, además se truncaron todas ellas y se aplicaron los distintos filtros que ofrecieron las diferentes bases de datos. En la segunda fase de la búsqueda bibliográfica se evaluaron las características psicométricas de los instrumentos seleccionados mediante las palabras claves validity, reliability, sensibility y reproducibility. Toda la estrategia de búsqueda bibliográfica se muestra en la figura 2 .

Criterios de inclusión. En la revisión se incluyeron los artículos publicados durante el 
Figura 2

Diagrama de flujo de la búsqueda bibliográfica y selección de artículos para incluir en la revisión

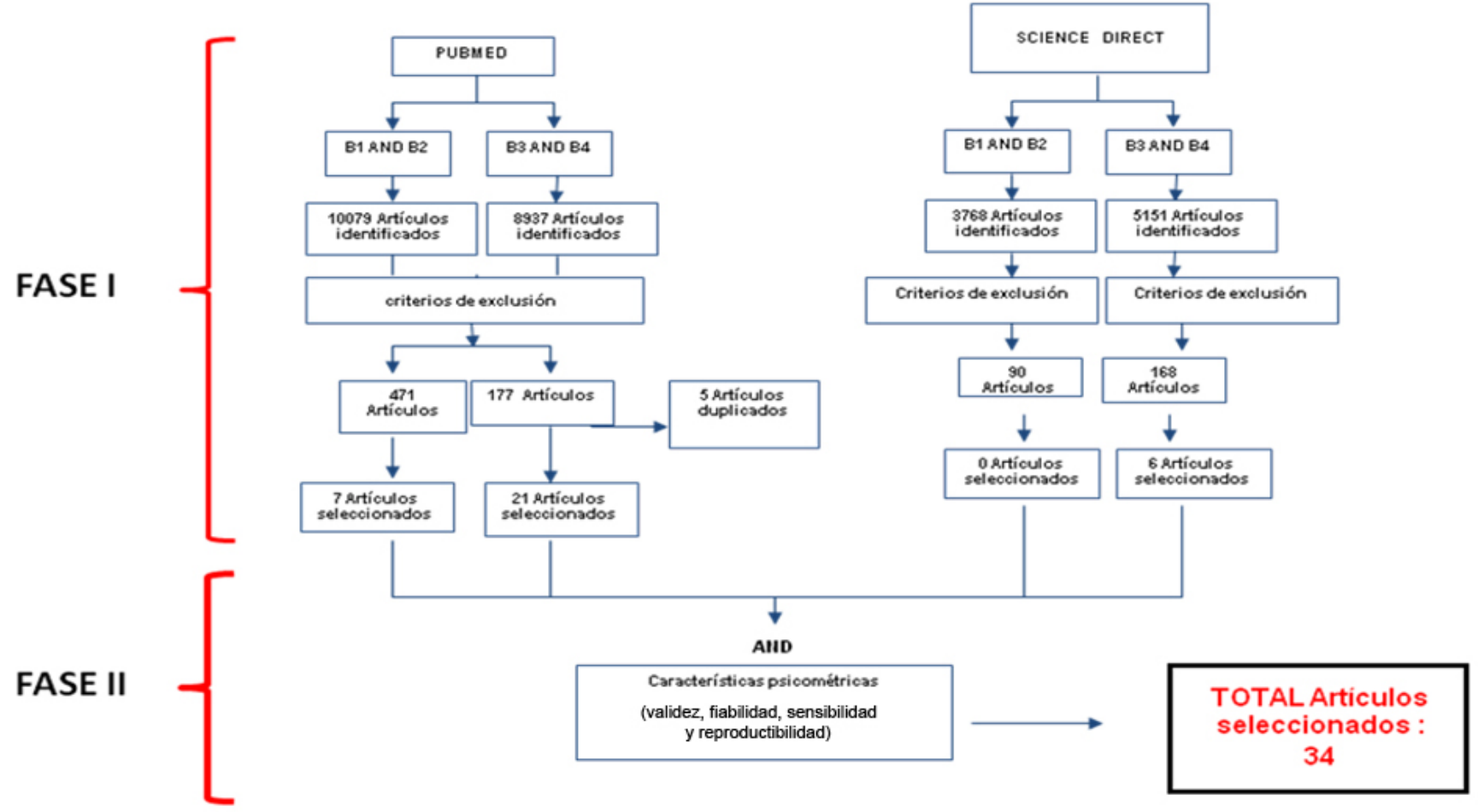

B 1: Mobility, movement, functional status, physical activity, physical function

B2: Mobility monitoring, mobility measures, scales, geriatric assessment y assessment

B3: Questionnaire, self report measures, accelerometer, accelerometry, pedometer, objective measures, doble-labeled water (DLW) B4: Geriatric, older people y elderly 
periodo de 2001 a 2013, salvo para los cuestionarios, ya que el análisis de las características psicométricas de algunos de ellos dataron de fechas que excedían el límite marcado y se incluyeron desde el año 1991. Respecto a los cuestionarios, se seleccionaron solo aquellos que se desarrollaron exclusivamente para personas mayores, aunque se incluyeron cuestionarios que en un principio se adaptaron a la población general como el Minesotta leisure time y IPAQ (versión corta) y que en posteriores versiones se adaptaron a la población mayor, obteniéndose así el IPAQ-E4 y la versión corta del Minesotta leisure time que se adaptó a personas mayores españolas ${ }^{14}$.

Criterios de exclusión. Se excluyeron los cuestionarios centrados en la población general y los que valoraban la disposición al cambio de conducta porque evaluaban variables o actividades de movilidad que no fueron propias de la población mayor y que podrían influir negativamente en los resultados de movilidad en este grupo de edad. No se incluyeron subescalas o cuestionarios que forman parte de otros cuestionarios que miden constructos más complejos.

Extracción de datos de los estudios primarios. La información que se extraería de cada artículo fue consensuada por los autores durante la fase de diseño del estudio y se centró en el análisis de la fiabilidad y/o validez del instrumento utilizado. La extracción de datos fue realizada por el primer autor y revisada por el resto de autoras durante el desarrollo del trabajo. Las discrepancias detectadas se resolvieron por consenso. La hoja de extracción de datos, basada en el protocolo Cochrane ${ }^{15}$, incluyó de cada artículo revisado: instrumento de medida de la movilidad, métodos de determinación y ventajas y desventajas, tipo de actividades, aplicación y análisis de la información.

Posteriormente se realizo un análisis narrativo de la información.

1. En primer lugar se agruparon los diferentes instrumentos según el método de deter- minación de movilidad en personas mayores: DLW, detectores de movimiento (podómetros y acelerómetros), medidas objetivas de la movilidad (funcionales) y cuestionarios. En ellos se analizaron las medidas obtenidas en cada uno así como las ventajas y desventajas.

2. En segundo lugar se realizó un análisis de los artículos referentes a los cuestionarios, que se agruparon en tres categorías: los que midieron movilidad, los que midieron actividad física y los que midieron el gasto energético. En cada uno de ellos se describieron sus principales características y se comparó su fiabilidad y validez.

\section{RESULTADOS}

El total de artículos seleccionados fueron 34 , de los que 23 correspondieron exclusivamente a cuestionarios $4,8,10,12-14,25,31-34,36-40,42-48,4$ a acelerómetros ${ }^{11,21-23}, 3$ a las medidas objetivas de la movilidad ${ }^{28-30}, 2$ artículos eran sobre podómetros ${ }^{19,20}$ y 2 para $\mathrm{DLW}^{18,26}$. Y 6 hacían referencia al análisis combinado de diferentes instrumentos ${ }^{16,17,24,27,35,41}$.

De los 34 estudios, 19 fueron estudios transversales $^{40,42,44,46-48,} 5$ longitudinales ${ }^{29,32,34,38,45}$ y 10 eran revisiones bibliográficas ${ }^{12,13,18-23,28,43}$. Todos ellos incluyeron información relativa a la fiabilidad y/o validez del instrumento de medida utilizado.

En la tabla 1 se refleja una síntesis de las ventajas e inconvenientes de los instrumentos que fueron seleccionados en este artículo.

Los 2 artículos ${ }^{18,26}$ que abordaron el agua doblemente marcada (DLW) coincidieron en señalar que este método determinó de forma exacta la energía gastada por los individuos a partir de la velocidad de producción de dióxido de carbono $\left(\mathrm{CO}_{2}\right)$ que fue hallada mediante 2 isótopos estables: deuterio $(2 \mathrm{H})$ y oxígeno-18 (180). La principal ventaja fue que el DLW fue el método más preciso para medir la movilidad, siendo el estándar de oro para el análisis de la validez de los instrumentos de movilidad. Sus principales limitaciones fue- 


\begin{tabular}{|c|c|c|c|}
\hline \multicolumn{4}{|c|}{$\begin{array}{c}\text { Tabla } 1 \\
\text { Instrumentos de medida de movilidad en personas mayores: } \\
\text { ventajas y desventajas }\end{array}$} \\
\hline Instrumentos de medida & Método de determinación & Ventajas & Desventajas \\
\hline Doble-labeled Water ${ }^{18-26}$ & $\begin{array}{l}\text { Energía empleada en la } \\
\text { realización de AF }\end{array}$ & $\begin{array}{l}\text { Mayor precisión } \\
\text { Gold Estándar }\end{array}$ & $\begin{array}{l}\text { Elevado precio materiales } \\
\text { Personal entrenado } \\
\text { Aplicabilidad en tamaños muestrales pequeños }\end{array}$ \\
\hline \multirow[b]{2}{*}{ Detectores de movimiento } & $\begin{array}{l}\text { Podómetros }{ }^{19-20} \\
\text { Registro número } \\
\text { de pasos dados }\end{array}$ & Fácil uso y colocación & $\begin{array}{l}\text { Menos precisión que acelerómetros } \\
\text { Dificultad para captar pasos cortos, movimientos lentos, } \\
\text { actividades de baja intensidad y actividades realizadas con } \\
\text { extremidades superiores } \\
\text { Falta de consenso sobre lugar de colocación }\end{array}$ \\
\hline & $\begin{array}{l}\text { Acelerómetros }{ }^{11,21-23} \\
\text { Frecuencia, duración } \\
\text { e intensidad de la AF, } \\
\text { estimando así el gasto } \\
\text { energético }\end{array}$ & $\begin{array}{l}\text { Mayor precisión que podóme- } \\
\text { tros }\end{array}$ & $\begin{array}{l}\text { Dificultad de seguimiento de protocolos de uso por parte } \\
\text { de las personas mayores } \\
\text { Elevado coste } \\
\text { Dificultad para captar pasos cortos, movimientos lentos, } \\
\text { actividades de baja intensidad y actividades realizadas con } \\
\text { extremidades superiores } \\
\text { Falta de consenso sobre lugar de colocación } \\
\text { Aplicabilidad en tamaños muestrales pequeños }\end{array}$ \\
\hline Medidas objetivas de la $\mathrm{AF}^{28-30}$ & $\begin{array}{l}\text { Valoración objetiva } \\
\text { del nivel de movilidad } \\
\text { de las personas }\end{array}$ & $\begin{array}{l}\text { Datos objetivos } \\
\text { Mayor precisión y sensibilidad } \\
\text { que los datos obtenidos en los } \\
\text { cuestionarios } \\
\text { Aplicabilidad en tamaños } \\
\text { muestrales grandes } \\
\text { Detección precoz de las altera- } \\
\text { ciones de la movilidad } \\
\text { Rápidos de realizar } \\
\text { Lenguaje sencillo y limitado } \\
\text { Coste no muy elevado } \\
\text { Fácil adaptación cultural }\end{array}$ & $\begin{array}{l}\text { Necesidad de equipamiento especifico } \\
\text { Necesidad espacio físico para su realización } \\
\text { Imposibilidad de realizarlo en personas con discapacidad } \\
\text { o enfermedades crónicas graves } \\
\text { Necesidad de entrevistadores más entrenados }\end{array}$ \\
\hline Cuestionarios ${ }^{4,8,10,12-14,25,31-34,36-40,42-48}$ & $\begin{array}{l}\text { Datos subjetivos sobre } \\
\text { el nivel de movilidad } \\
\text { de los participantes }\end{array}$ & $\begin{array}{l}\text { Baratos, sencillos y fáciles de } \\
\text { utilizar } \\
\text { No requieren equipamiento adi- } \\
\text { cional } \\
\text { Se pueden realizar por un entre- } \\
\text { vistador, por teléfono o autoad- } \\
\text { ministrados }\end{array}$ & $\begin{array}{l}\text { Datos subjetivos } \\
\text { Menos precisos y sensibles que las medidas objetivas } \\
\text { Adaptación cultural más compleja que las medida objetivas } \\
\text { Resultados influenciados por percepciones subjetivas de los } \\
\text { individuos, problemas cognitivos y memoria, niveles educa- } \\
\text { tivos bajos y por la tendencia de los encuestados a responder } \\
\text { lo socialmente correcto }\end{array}$ \\
\hline
\end{tabular}


ron el coste tan elevado de los isotopos empleados, el equipo tan especializado que precisó y además que no informaron ni del tipo ni de la duración de la movilidad evaluada ${ }^{12}$ 16,18,25,26. Ello limitó el uso del DLW a muestras de pequeño tamaño.

Los artículos que estudiaron los detectores de movimiento, podómetros ${ }^{19,20}$ y acelerómetros ${ }^{11,21-23}$, definieron estos instrumentos como capaces de captar de diferente manera la cantidad de movimiento realizado por las personas mediante un sistema que se fija en una parte del cuerpo.

Los podómetros midieron el número de pasos dados mediante un sistema pendular pero no la intensidad de la $\mathrm{AF}^{27} \mathrm{y}$ fueron capaces de medir dos tipos de actividad: andar y correr. Los artículos señalaron como principales ventajas que fueron unos excelentes instrumentos para medir la AF, de hecho se correlacionaron positivamente con otros instrumentos de medición de la AF, como acelerómetros, DLW donde la correlación fue moderada y con cuestionarios sobre movili$\operatorname{dad}^{16,20,27}$. Fueron una herramienta sencilla y fácil de usar por las personas mayores, además de no interferir en sus hábitos de vida y tener un coste muy bajo ${ }^{16}$. La gran desventaja de estos instrumentos fue su escasa precisión para captar y cuantificar la movilidad en personas que realizaron movimientos lentos o dieron pasos $\operatorname{cortos}^{27}$, como fue el caso de las personas mayores. Además, este grupo de edad tuvo una actitud sedentaria durante un gran número de horas al día, dificultándose aun más la captación de la AF y limitando por ello la validez de este instrumento de medida en las personas mayores.

Los acelerómetros miden la magnitud de los cambios de la aceleración del centro de masas del cuerpo durante el movimiento. Al medirse la aceleración se obtiene una medición más precisa de la intensidad, la frecuencia y la duración de la actividad que permitió cuantificar el movimiento de las personas y determinar el gasto energético ${ }^{19,20}$. La medida de la AF fue realizada mediante sensores eléctricos que determinaron el movimiento en diferentes dimensiones. Los detectores de movimiento podían ser uniaxiales, biaxiales o triaxiales, siendo los acelerómetros triaxiales los que aportaron las valoraciones más precisas de la $\mathrm{AF}^{16,21-23}$. Como ventajas, los acelerómetros son detectores de movimientos más exactos que los podómetros para evaluar la movilidad en las personas mayores ${ }^{16,21}$, dicha afirmación fue corroborada por Gardner et al. tras obtener una alta correlación con el $\mathrm{DLW}^{16}$. Entre las limitaciones, como sucedía con el podómetro, están que los acelerómetros presentaron dificultad para detectar toda la energía realizada por las personas mayores, ya que realizan actividades de baja intensidad y actividades que implican principalmente las extremidades superiores, y permanecen largos periodos de tiempo en actitud sedentaria, disminuyendo todo ello la precisión de estos instrumentos ${ }^{16}$. Otra limitación que se planteó en este grupo de edad fueron los problemas en la comprensión del protocolo de utilización de estos instrumentos debido a sus dificultades cognitivas ${ }^{21}$.

Entre las pruebas objetivas de la movilidad se encontraron el Short Physical Performance Battery (SPPB), Sit and Stand, Time Up \& Go, Gait Speed 400 meters (test 400 meters walk), Alternative Step o el Stair ascent and descent entre otros ${ }^{17,28-30}$. La aplicación de estos instrumentos consistió en la realización, por parte de los individuos, de una serie de tareas específicas que se repitieron un número determinado de veces. Estas actividades fueron observadas por un entrevistador que realizó una evaluación objetiva de la movilidad en función del número de repeticiones o del tiempo empleado en realizarlas ${ }^{17,28-30}$. Una de las grandes ventajas de estas pruebas fue que permitieron detectar precozmente e incluso in situ la presencia de problemas en la movilidad $^{17,28}$ y además que estos instrumentos tuvieron una adecuada precisión y fiabilidad test-retest para medir la movilidad ${ }^{17}$. Además, aportaron datos objetivos de la movilidad que fueron más precisos y más sensibles al cambio que los datos de los cuestionarios. Entre 
las limitaciones figuran su compleja administración, la necesidad de un entrevistador entrenado y de instalaciones adecuadas y una interpretación clínica más compleja $a^{17,28,29,32}$. Por ello fueron escasos los estudios que emplearon este instrumento para medir la movilidad en las personas mayores. El más utilizado fue el Short Physical Performance Battery $(\mathrm{SPPB})^{8,31}$, validado en población española mayor de 70 años ${ }^{49}$.

Los artículos que analizaban cuestionarios de AF y movilidad fueron los más numerosos y se desarrollaron para todo tipo de grupos de edad y entornos culturales. Así Ainsworth et al ${ }^{12}$ llegaron a describir hasta 39 diferentes, de los cuales solo el Minnesota Leisure Time Physical Activity, Yale Physical Activity (YPAS) y el Modified Baecke Questionnaire fueron validados para población española ${ }^{12}$. El 30,7\% de los cuestionarios encontrados midieron la movilidad de las personas mayores de 65 años, este porcentaje fue muy bajo si tenemos en consideración que las personas mayores son el grupo poblacional que más problemas de movilidad presentan ${ }^{14,21}$.

En las tablas 2,3 y 4 se presenta una descripción de los 15 cuestionarios de movilidad.

Según el tipo de medida se clasifican en: los que midieron propiamente movilidad son: LSA $^{9,35}$, MAT-SF ${ }^{31,32,38, ~ Z U T P H E N ~ 12,40, ~ 45,46 ~}$ (tabla 2), los que midieron actividad física IPAQ-E ${ }^{4}$, LASA PAQ ${ }^{12,34}$, Minesotta Leisure Time $e^{12,14}$, Modified Baecke $e^{12,25,33,34}$, PASE ${ }^{10,12,16}$, Physical Fitness and Exercise Activity of Older Adult Scale (PFE) ${ }^{12,39}$, RAPA ${ }^{12,43,47}$, TAPA $^{44}$ (tabla 3), los que midieron gasto energético CHAMPS ${ }^{10,12,16}$, QAPSE ${ }^{12,36}$, YPAS ${ }^{10,12,16,37}$, PRISCUS-PAQ ${ }^{40-42}$ (tabla 4).

Respecto al tipo de actividades, 10/15 cuestionarios evaluaron actividades domésticas como tal, de los cuales 5 incorporaron además actividades de ocio y tiempo libre.

Según el tipo de información, los cuestionarios que midieron la movilidad propiamente dicha la evaluaron mediante preguntas sobre patrones o niveles de movilidad mientras que los que midieron $\mathrm{AF}$ y gasto energético lo hicieron mediante preguntas sobre la frecuencia y duración de diferentes actividades domésticas, de ocio y de ejercicio, así como mediante actividades de diferentes grados de intensidad realizadas durante el último mes o semana (excepto el Minesotta Leisure Time cuyo tiempo de recuerdo de las actividades es del último año).

Teniendo en cuenta la valoración del cuestionario, los que midieron movilidad propiamente dicha clasificaron a los sujetos en diferentes niveles y el resto (5/15) utilizaron alguna escala para clasificar a los sujetos.

\begin{tabular}{|c|c|c|c|c|c|}
\hline \multicolumn{6}{|c|}{$\begin{array}{c}\text { Tabla } 2 \\
\text { Características de los cuestionarios que miden }\end{array}$} \\
\hline Cuestionario & $\begin{array}{c}\text { Tipo } \\
\text { de actividades }\end{array}$ & $\begin{array}{c}\text { Tipo } \\
\text { de información }\end{array}$ & Valoración & $\begin{array}{c}\text { Horizonte } \\
\text { temporal }\end{array}$ & $\begin{array}{c}\text { Recogida } \\
\text { de información }\end{array}$ \\
\hline $\operatorname{LSA}^{9,35}$ & $\begin{array}{l}\text { Actividades de las } \\
\text { personas en unas de- } \\
\text { terminadas aéreas de } \\
\text { su casa, vecindario y } \\
\text { ciudad. }\end{array}$ & $\begin{array}{c}\text { Evalúa } \\
\text { los patrones d } \\
\text { e movilidad } \\
\text { de las personas }\end{array}$ & $\begin{array}{c}\text { Puntuación } \\
\text { de } 0 \text { a } 120 \\
\text { a mayor puntuación } \\
\text { mayor rango } \\
\text { de movilidad }\end{array}$ & $1 \mathrm{mes}$ & $\begin{array}{c}\text { Entrevista } \\
\text { o vía telefónica } \\
\text { (para test-retest) }\end{array}$ \\
\hline MAT-sf $31,32,38$ & $\begin{array}{c}\text { Caminar con diferente } \\
\text { velocidad, inclinación } \\
\text { y su realización con o } \\
\text { sin cargas }\end{array}$ & $\begin{array}{c}\text { Nivel } \\
\text { de movilidad }\end{array}$ & $\begin{array}{l}\text { Da una puntuación, } \\
\text { a mayor puntuación } \\
\text { mayor movilidad }\end{array}$ & - & $\begin{array}{l}\text { Video } \\
\text { más entrevista } \\
\text { personal } \\
(5 \mathrm{~min})\end{array}$ \\
\hline ZUTPHEN $^{12,40,45,46}$ & $\begin{array}{l}\text { Caminar, montar en } \\
\text { bici, jardinería, traba- } \\
\text { jos caseros, deportes y } \\
\text { de ocio } 7,20,21 \text { (solo } \\
\text { en hombres) } \\
\text { Además tareas domés- } \\
\text { ticas en mujeres }^{33}\end{array}$ & $\begin{array}{l}\text { Frecuencia } \\
\text { y duración } \\
\text { de las } \\
\text { actividades } \\
\text { realizadas }\end{array}$ & Activo y no activo & 1 semana & Entrevista \\
\hline
\end{tabular}




\begin{tabular}{|c|c|c|c|c|c|}
\hline \multicolumn{6}{|c|}{$\begin{array}{c}\text { Tabla } 3 \\
\text { Características de los cuestionarios que miden actividad física en personas mayores }\end{array}$} \\
\hline Cuestionario & $\begin{array}{c}\text { Tipo } \\
\text { de actividades }\end{array}$ & $\begin{array}{c}\text { Tipo } \\
\text { de información }\end{array}$ & Valoración & $\begin{array}{l}\text { Horizonte } \\
\text { temporal }\end{array}$ & $\begin{array}{l}\text { Recogida } \\
\text { de } \\
\text { información }\end{array}$ \\
\hline IPAQ-E $^{4}$ & $\begin{array}{l}\text { Actividades moderadas } \\
\text { y vigorosas, sentarse y } \\
\text { caminar }\end{array}$ & $\begin{array}{c}\text { Frecuencia y duración } \\
\text { de la actividad física }\end{array}$ & - & $\begin{array}{l}\text { Últimos } \\
7 \text { días }\end{array}$ & Entrevista \\
\hline LASA-PAQ $^{12,34}$ & $\begin{array}{l}\text { Caminar, montar en } \\
\text { bicicleta, jardinería, tareas } \\
\text { domésticas ligeras e } \\
\text { intensas y un máximo de } \\
\text { dos deportes }\end{array}$ & $\begin{array}{c}\text { Cuantificación } \\
\text { de la AF }\end{array}$ & - & 2 semanas & $\begin{array}{l}\text { Entrevista } \\
\text { (3-9 min) }\end{array}$ \\
\hline $\begin{array}{l}\text { Minessota leisure } \\
\text { times } 1^{2,14}\end{array}$ & $\begin{array}{l}\text { Actividades de ocio, do- } \\
\text { mésticas, caminar bailar, } \\
\text { subir escaleras y trabajar } \\
\text { en el huerto }\end{array}$ & $\begin{array}{c}\text { Calculo de los MET } \\
\text { (gasto calórico basal) } \\
\text { según frecuencia } \\
\text { y duración de la AF }\end{array}$ & $\begin{array}{c}\text { Clasifica en muy } \\
\text { activos, activos, } \\
\text { moderadamente } \\
\text { activos } \\
\text { y sedentarios }^{43}\end{array}$ & \begin{tabular}{|c|}
1 año \\
versión original \\
y su validación \\
en español 18-61 \\
años ${ }^{12,43}$ ) \\
1 semana o 1 \\
mes para la \\
versión corta \\
validada para \\
los ancianos \\
españoles ${ }^{43}$
\end{tabular} & Entrevista \\
\hline Moedified Baecke $e^{12,25,33,34}$ & $\begin{array}{l}\text { Domésticas, deportivas y } \\
\text { de ocio }\end{array}$ & AF diaria & $\begin{array}{l}\text { Clasificación } \\
\text { en alta, baja } \\
\text { o moderada AF }\end{array}$ & Último año & $\begin{array}{l}\text { Entrevista } \\
15 \text { minutos }\end{array}$ \\
\hline PASE $^{10,12,16}$ & $\begin{array}{l}\text { Actividades domésticas, } \\
\text { de ocio o deportivas, } \\
\text { laborales, ocupacionales } \\
\text { remuneradas o no, y otras }\end{array}$ & $\begin{array}{c}\text { Frecuencia y duración } \\
\text { de las actividades } \\
\text { realizadas para calcular } \\
\text { la AF total }\end{array}$ & 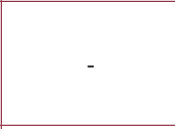 & $\begin{array}{l}\text { Últimos } \\
7 \text { días }\end{array}$ & $\begin{array}{c}\text { Autoadministra- } \\
\text { do, vía telefó- } \\
\text { nica o mediante } \\
\text { entrevista } \\
(5 \mathrm{~min}) \\
\end{array}$ \\
\hline $\begin{array}{l}\text { Physical Fitness And } \\
\text { Exercise Activity Of } \\
\text { Older Adult Scale }\end{array}$ & $\begin{array}{l}\text { Actividades físicas } \\
\text { ocupacionales y de } \\
\text { recreo, y barreras para su } \\
\text { realización }\end{array}$ & Niveles de AF & - & - & Entrevista \\
\hline RAPA $^{12,43}$ & $\begin{array}{l}\text { Actividades ligeras, } \\
\text { moderadas e intensas, } \\
\text { evaluando además fuerza } \\
\text { y flexibilidad }\end{array}$ & Niveles de AF & $\begin{array}{c}\text { Cinco niveles } \\
\text { de AF, desde } \\
\text { sedentario hasta } \\
\text { activo } \\
\end{array}$ & 1 semana & $\begin{array}{l}\text { Autoadministrado } \\
\text { (2 min })\end{array}$ \\
\hline TAPA $^{44}$ & $\begin{array}{l}\text { Evalúa ocio, trabajo y } \\
\text { actividades que implican } \\
\text { movimiento, fuerza y } \\
\text { flexibilidad. }\end{array}$ & Niveles de AF & $\begin{array}{l}\text { Cuatro niveles } \\
\text { de AF, desde } \\
\text { sedentario hasta } \\
\text { activo }\end{array}$ & 1 semana & Telefónica \\
\hline
\end{tabular}

El horizonte temporal o intervalo de tiempo al que hacían referencia los cuestionarios de movilidad fue muy variable, con un rango de una semana hasta un año. La recogida de información en 12 de15 de ellos fue a través de entrevista directa, la duración de la cumplimentación se situó entre los 2 a 20 minutos, el RAPA fue el de menor tiempo de administración y el YPAS el de mayor (30 minutos) $)^{12,43,44,47}$.

Las propiedades psicométricas evaluadas en estos cuestionarios fueron la fiabilidad, la validez y la sensibilidad, cuyos resultados pueden observarse en las tablas 5,6 y 7 en las que se agruparon también según medían la movilidad, la actividad física o el gasto energético.
Respecto a la fiabilidad, solo tres de los cuestionarios incluyeron la consistencia interna mediante la $\alpha$ de Cronbach (Modified Baecke, PASE y PFE), que osciló de 0,96 a 0,69 , lo que mostró una buena consistencia interna. La fiabilidad a través del test-retest, se estudió en todos los cuestionarios excepto en TAPA e IPAQ-E. Los valores de sus coeficientes de Spearman oscilaron entre $0,997 \mathrm{p}<0,0001$ para QAPSE12 y de 0,556 $\mathrm{p}<0,0001$ para $\mathrm{PFE}^{12}$. La fiabilidad intraobservador sólo se analizó en el cuestionario LASA-PAQ y tuvo un índice de Kappa de $0,65-0,75 \mathrm{p}<0,01^{12,34}$.

En relación a la validez, los cuestionarios realizaron el estudio de la validez de criterio, de constructo o de ambas. Todos los cuestio- 


\section{Tabla 4}

\begin{tabular}{|c|c|c|c|c|c|}
\hline \multicolumn{6}{|c|}{$\begin{array}{c}\text { Tabla } 4 \\
\text { Características de los cuestionarios que miden gasto energético en personas mayores }\end{array}$} \\
\hline Cuestionario & Tipo de actividades & Tipo de información & Valoración & Horizonte temporal & $\begin{array}{c}\text { Recogida } \\
\text { de información }\end{array}$ \\
\hline CHAMPS ${ }^{10,12,16}$ & $\begin{array}{l}\text { Actividades domésticas, de ocio y ejercicio físico, } \\
\text { de intensidad variable (ligera moderada e intensa) }\end{array}$ & $\begin{array}{l}\text { Gasto calórico semanal } \mathrm{Kcal} / \mathrm{semana} \\
\text { y la frecuencia de todas las actividades }\end{array}$ & - & $\begin{array}{l}\text { Una semana típica } \\
\text { del último mes }\end{array}$ & $\begin{array}{c}\text { Autoadministrado, } \\
\text { por vía telefónica } \\
\text { o mediante entrevista } \\
(10-15 \mathrm{~min})\end{array}$ \\
\hline QAPSE $^{12,36}$ & Actividades domésticas, ocio, trabajo y actividades básicas & $\begin{array}{l}\text { Gasto energético medio diario habitual } \\
\text { y por actividades ( MET/día y Kcal/día). } \\
\text { Frecuencia y duración de la actividad }\end{array}$ & - & 1 Semana & $\begin{array}{l}\text { Autoadministrado } \\
\text { (20 min) } \\
\text { Entrevista } \\
(30 \mathrm{~min})\end{array}$ \\
\hline YPAS ${ }^{10,12,16,37}$ & $\begin{array}{l}\text { Dos secciones, la } 1^{\text {a }} \text { evalúa tiempo trabajo domés- } \\
\text { tico, jardinería, cuidado de familiares, actividades } \\
\text { deportivas y de ocio; la } 2^{\mathrm{a}} \text { frecuencia y duración de } \\
\text { actividades intensas, y pasear, movimiento, perma- } \\
\text { necer de pie y sentado. } \\
\text { Versión validada en español evalúa las misma ac- } \\
\text { tividades. }\end{array}$ & $\begin{array}{l}\text { El tiempo total de las actividades evaluadas, } \\
\text { el gasto calórico en } \mathrm{Kcal} / \mathrm{min} 107 \mathrm{y} \text { las di- } \\
\text { mensiones de actividades } 14\end{array}$ & - & $\begin{array}{l}\text { Una semana típica } \\
\text { del último mes para la } 1^{\mathrm{a}} \\
\text { y } 2^{\mathrm{a}} \text { sección } 10,12 \\
\text { Una semana típica } \\
\text { para la } 1^{\mathrm{a}} \text { sección } \\
\text { y el mes pasado } \\
\text { para la } 2^{\mathrm{a}} \text { sección } 14\end{array}$ & $\begin{array}{l}\text { Entrevista } 1^{0,14} \\
\text { y por vía } \\
\text { telefónica } \\
(20 \mathrm{~min})\end{array}$ \\
\hline PRISCUS-PAQ ${ }^{40,41,42}$ & Actividades domésticas, deportivas e inactividad & Gasto energético semanal consumida por la AF & $\begin{array}{l}\text { Puntuación } \\
\text { total }\end{array}$ & 1 semana & Telefónica \\
\hline
\end{tabular}

\begin{tabular}{|c|c|c|c|c|c|}
\hline & Propied & dades psicométri & icas de los & $\begin{array}{l}\text { Tabla } 5 \\
\text { cuestionarios que miden movilidad en personas mayores }\end{array}$ & \\
\hline & & iabilidad & & Validez & Sensibilidad \\
\hline Cuestionario & $\begin{array}{c}\text { Consistencia } \\
\text { interna }\end{array}$ & Test-retest & Criterio & Constructo & - \\
\hline LSA $^{9,35}$ & - & $\begin{array}{l}\mathrm{ICC}=0,86-0,96 \\
\quad(2 \text { semaas })^{9}\end{array}$ & 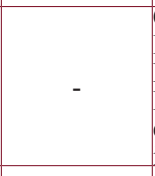 & $\begin{array}{l}\text { Correlación significativa con una serie de medidas de salud física y mental (SPPB, } \\
\text { IDL, ADL, SF12, GDS, condiciones de comorbilidad y autoreporte de la salud), } \\
\text { Dichas correlaciones son mayores entre el LSA y las funciones físicas que entre el } \\
\text { LSA y las funciones mentales, Además, las correlaciones más significativa son entre } \\
\text { el LSA y el SPBB, ADL, IDL y el GDS p }<0,001, \text { que entre el LSA y el resto de } \\
\text { instrumentos mencionados anteriormente } p<0,01^{9,35}\end{array}$ & - \\
\hline MAT-sf $31,32,38$ & 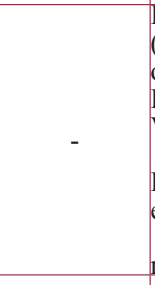 & $\begin{array}{l}I C C=0,93 \mathrm{p}<0,0001 \\
\text { ( } 2 \text { semanas) Estudio }^{3 \text { original }^{31,32}} \\
\text { Estudio de } \\
\text { Validación en } \\
\text { Portugués y } \\
\text { Español } \\
\text { en latino América } \\
\text { ICC }=0,94 \text { y } 0,81 \\
\text { respectivamente }\end{array}$ & 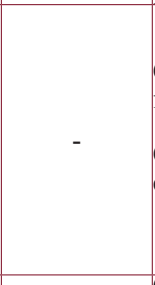 & $\begin{array}{l}\text { Correlaciones significativas con } 400 \text { meters walk test, PAT-D (Pepper assessment toll } \\
\text { for disability) y SPPB, hallándose en todas ellas } \mathrm{p}<0,001^{31,32} \\
\text { Correlaciones significativas con todas las dimensiones del SPPB tanto en Brasil } \\
\text { como en Colombia } p<0,001^{38}\end{array}$ & - \\
\hline ZUTPHEN ${ }^{12,40,45,46}$ & - & $\begin{array}{l}\mathrm{S}=0,96 \\
(4 \text { meses })^{12,45,46}\end{array}$ & $\begin{array}{l}\text { Correlación } \\
\text { con DLW } \\
\mathrm{S}=0,61 \\
\mathrm{p}<0,001^{12,46}\end{array}$ & $\begin{array}{l}\text { Correlación moderada con podómetro } \mathrm{p}=0,36 \mathrm{p}<0,001 \text { con el cuestionario de Zutphen } \\
\text { modifificado (introducidas tareas domesticas para evaluar mujeres ancianas) } \\
\text { Correlación moderada con Zutphen sin modificar con el acelerómetro } \mathrm{p}=0,35 \mathrm{p}<0,001^{40}\end{array}$ & - \\
\hline
\end{tabular}




\begin{tabular}{|c|c|c|c|c|c|}
\hline \multirow[b]{3}{*}{ Cuestionario } & dades psice & ométricas de los cuesti & $\begin{array}{l}\text { Tabla } 6 \\
\text { tionarios que miden activi }\end{array}$ & idad física en personas mayores & \multirow[b]{3}{*}{ Sensibilidad } \\
\hline & \multicolumn{2}{|c|}{ Fiabilidad } & \multicolumn{2}{|c|}{ Validez } & \\
\hline & $\begin{array}{c}\text { Consistencia } \\
\text { interna }\end{array}$ & Test-retest & Criterio & Constructo & \\
\hline $\mathrm{IPAQ} \mathrm{E}^{4}$ & 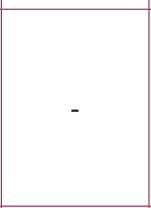 & - & $\begin{array}{l}\text { Correlación significativa de todos } \\
\text { los dominios del IPAQ-E con acele- } \\
\text { rómetro } \\
\mathrm{S}=0,277-0,471 \\
\text { Correlación entre los niveles de } \\
\text { actividad física del IPAQ-E (leve, } \\
\text { moderado e intenso ) con Proteína C } \\
\text { reactiva P (ANOVA) }=0,041\end{array}$ & $\begin{array}{l}\text { Especificidad del } 85 \% \text { para identificar a } \\
\text { participantes de baja intensidad y sensibilidad } \\
\text { del } 81 \% \text { para identificar a los participantes más } \\
\text { activos. }\end{array}$ & - \\
\hline LASA-PAQ ${ }^{12,34}$ & - & $\begin{array}{l}\text { Correlación altamente signifi- } \\
\text { cativa con } 7 \mathrm{~d} \text { physical activity } \\
\text { recall } \mathrm{S}=0,68 \mathrm{p}<0,001 \text {, y co- } \\
\text { rrelación moderada con el podó- } \\
\text { metro } \mathrm{S}=0,56 \mathrm{p}<0,0011^{12,34}\end{array}$ & - & $\begin{array}{l}\text { Correlación significativa con medidas del des- } \\
\text { empeño } \mathrm{S}=0,44 \text {, fuerza del apretón de mano } \\
\mathrm{S}=0.33 \text { y con fuerza de la pierna dominante } \\
\mathrm{S}=0.28 \text {. Todas ellas } \\
\mathrm{p}<0.0011^{2,34}\end{array}$ & - \\
\hline Minessota Leisure Times ${ }^{12,14}$ & - & $\begin{array}{l}\text { Muestra correlación con DLW } \\
\text { en actividades moderadas e } \\
\text { intensas } \mathrm{S}=0,5 \text { y } 0,47 \text { respec- } \\
\text { tivamente p }<0,05 \text { y con VO- } \\
2 \text { max (versión original) } \\
\text { Correlación entre la versión } \\
\text { corta en ancianos españoles con } \\
\text { la versión original de este cues- } \\
\text { tionario Kappa }=0,93^{14}\end{array}$ & - & $\mathrm{f}$ & - \\
\hline Moedified Baecke ${ }^{12,25,33}$ & $0,96^{12,33}$ & $\mathrm{~S}=0,89^{12,33} \quad(3$ meses $)$ & $\begin{array}{l}\text { No muestra correlación con } \\
\mathrm{VO}_{2} \text { max } 12 \text {, pero si una correlación } \\
\text { moderada con DLW S }=0,54^{33}\end{array}$ & - & - \\
\hline PASE ${ }^{10,12,16}$ & $0,694^{12}$ & $\begin{array}{c}\text { Autoadminsitrado } \begin{array}{l}\mathrm{S}=0,84 \\
\left(^{(3-7 \text { semanas })^{10,12,16}}\right. \\
\text { Vía telefónica } S=0,75^{10,12} \\
\text { Entrevista } S=0,68 \\
\quad(3-7 \text { semanas })^{16}\end{array}\end{array}$ & $\begin{array}{l}\text { No muestra correlación con DLW ni } \\
\text { con VO2max 10,12,Sin embargo, } \\
\text { Schuit et all observaron que la actividad } \\
\text { medida por el PASE se correlaciono po- } \\
\text { sitivamente con DLW } S=0,58^{16} \\
\text { Correlación entre PASE y la AF medida } \\
\text { durante tres días por acelerómetro } S= \\
0,64 \text { y } 0,49 \text { para la muestra y submues- } \\
\text { tra respectivamente }{ }^{16} \\
\text { Correlación significativa entre } \\
\text { el PASE y el YPAS y el CHAMPS } \mathrm{p}= \\
0,61 \text { y } 0,58 \text { respectivamente } \\
\text { p }<0,0001^{10}\end{array}$ & $\begin{array}{l}H_{0} \text { : Los participantes de los centros comunitarios } \\
\text { desarrollan mayor actividad física que los ingre- } \\
\text { sados en residencias de ancianos }<<0,0001^{12} \\
\text { Correlación moderada con salud general, ritmo } \\
\text { cardiaco, fuerza de apretón de manos y de la } \\
\text { pierna dominante, y correlación baja pero signi- } \\
\text { ficativa con edad consumo de oxigeno, presión } \\
\text { sistólica y puntuación en la escala de equilibrio } \\
\text { de Berg }{ }^{10,12} \\
\text { Correlación significativa con medidas del des- } \\
\text { empeñño EPESE, podómetro, SF } 36,6 \text { min Walk } \\
\text { p }<0,01^{10,12} \text {, Mini log ankle y mini log waste } \\
\text { p }<0,001^{10} \text {. }\end{array}$ & $\begin{array}{l}\text { Sensibilidad al cam- } \\
\text { bio tras estudio pilo- } \\
\text { to de intervención de } \\
\text { asesoramiento médi- } \\
\text { co de } 6 \text { semanas de } \\
\text { duración }{ }^{10,12}\end{array}$ \\
\hline
\end{tabular}


Tabla 7

Propiedades psicométricas de los cuestionarios que miden gasto energético en personas mayores

\begin{tabular}{|c|c|c|c|c|c|}
\hline \multirow[b]{2}{*}{ Cuestionario } & \multicolumn{2}{|r|}{ Fiabilidad } & \multicolumn{2}{|r|}{ Validez } & \multirow[b]{2}{*}{ Sensibilidad } \\
\hline & $\underset{\text { interna }}{\text { Consistencia }}$ & Test-retest & Criterio & Constructo & \\
\hline CHAMPS $^{10,12}$ & - & $\begin{array}{c}\text { ICC } 0,62 \\
\text { para todas las actividades } \\
(2 \text { semanas })^{10} \\
\text { IC }=0,6612 \\
\text { para todas las actividades }\end{array}$ & $\begin{array}{c}\text { Correlación altamente significativa con } \\
\text { cuestionarios PASE e YPAS p }=0,58 \text { y } \\
0,64 \text { respectivamente } \\
\mathrm{p}<0,0001^{10}\end{array}$ & $\begin{array}{l}\text { Se correlaciona con medidas EPESE, } 6 \text { Min } \\
\text { walk, SF } 36 \text {, podómetro }{ }^{10,12} \text {, Mini-Log ankle y } \\
\text { Mini Log Waist }{ }^{10} p<0,01 \text { en todas excpeto Mini } \\
\text { Log Waist } \mathrm{p}<0,001, \\
\text { Contraste de hipótesis en grupo de actividad } \\
\text { conocida } \mathrm{p}<0,0001^{12} \\
\mathrm{H}_{0} \text { : Los participantes de los centros comuni- } \\
\text { tarios desarrollan mayor actividad física que los } \\
\text { ingresados en residencias de ancianos } \mathrm{p}<0,0001^{12}\end{array}$ & $\begin{array}{l}\text { La sensibilidad al cambio } \\
\text { fue moderada para las } \\
\text { medidas de las frecuencias } \\
\text { y bajo- moderado para el } \\
\text { gasto calórico }{ }^{12} \\
\text { Este cuestionario ha } \\
\text { demostrado sensibilidad al } \\
\text { cambio después de } 6 \text { y } 12 \\
\text { meses de un programa de } \\
\text { promoción de la actividad } \\
\text { física en ancianos, y } \\
\text { después de } 12 \text { meses de } \\
\text { un programa de resistencia } \\
\text { y fortalecimiento para las } \\
\text { comunidades de ancianos }{ }^{10}\end{array}$ \\
\hline QAPSE $^{12,36}$ & - & $\begin{array}{c}\mathrm{S}=0,997 \mathrm{p}<0,0001 \\
(6 \text { semanas })^{12} \\
\text { Gasto energético diario } \\
\mathrm{S}=0,997 \\
\text { e índice de movimiento } \\
\mathrm{S}=0,648, \text { siendo para ambos } \\
\mathrm{p}<0,0001 \\
(6 \text { semanas })^{12} \\
\end{array}$ & $\begin{array}{l}\text { Correlación con VO2 max con gasto } \\
\text { energético diario } \mathrm{S}=0,56 \mathrm{p}<0,0001^{12,36} \\
\begin{array}{l}\text { Correlación entre la actividad deportiva } \\
\text { y el DLW } \mathrm{S}=0,54 \mathrm{p}<0,05^{12}\end{array}\end{array}$ & $\begin{array}{l}\text { Correlación con la ingesta calórica y nivel de } \\
\text { actividad } \mathrm{S}=0,792 \mathrm{y}-0,43 \text { respectivamente. } \\
\text { Ambas } \mathrm{p}<0,0001^{12} \\
\begin{array}{l}\text { Gasto energético diario correlacionado positiva- } \\
\text { mente con el peso corporal } \mathrm{S}=0,464, \text { masa grasa } \\
\text { libre } \mathrm{S}=0,639 \text { y correlacionado negativamente con } \\
\text { el porcentaje de grasa corporal } \mathrm{S}=0,501^{36}\end{array}\end{array}$ & - \\
\hline PRISCUS-PAQ $^{41,42}$ & - & $\begin{array}{c}\text { ICC total } 0,59 \\
\text { ICC caminar } 0,47, \\
\text { ICC montar bicicleta } 0,82, \\
\text { ICC Tareas del hogar } 0,59 \text { ICC } \\
\text { Act deportivas } 0,76^{42} \\
\end{array}$ & $\begin{array}{l}\text { Correlación entre este cuestionario } \\
\text { y el acelerómetro } S=0,28^{41}\end{array}$ & ( & - \\
\hline YPAS $^{10,12,16,37}$ & y & $\begin{array}{c}\text { Tiempo total } S=0,57 \\
\text { y para gasto energético semanal } \\
\mathrm{S}=0,58, \text { para ambos } \mathrm{p}<0,0001 \\
(2 \text { semanas })^{10,12,16} \\
\text { Versión validada en español } \\
\text { Tiempo y para gasta energé-- } \\
\text { tico semanal } \mathrm{p}=0,66 \text { y } 0,65 \\
\mathrm{p}<0,001^{37}\end{array}$ & 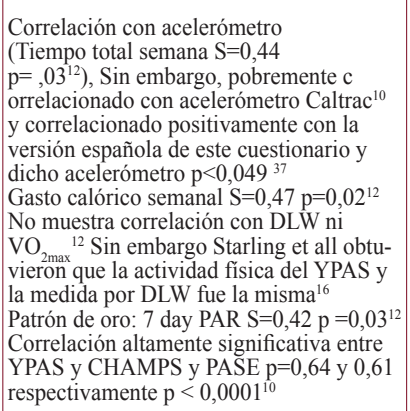 & $\begin{array}{l}\text { El gasto energético semanal de este cuestionario } \\
\text { fue correlacionado moderadamente con la presión } \\
\text { diastólica } \mathrm{p}<0,001^{10,12} \\
\mathrm{H}_{0} \text { : Los participantes de los centros comunitarios } \\
\text { desarrollan mayor actividad física que los ingresa- } \\
\text { dos en residencias de ancianos } \mathrm{p}<0,0001^{12} \\
\text { Correlación significativa con medidas del desem- } \\
\text { peño EPESE, podómetro, } 6 \text { min walk y SF } 36 \mathrm{p} \\
<0,01^{10,12}, \text { Mini log ankle y mini log waist } \mathrm{p}< \\
0,001^{10} \\
\end{array}$ & $\begin{array}{l}\text { Sensibilidad al cambio } \\
\text { tras actuar en un grupo de } \\
\text { intervención durante } 12 \\
\text { semanas se incremento } \\
\text { AF ( excepto en estar sen- } \\
\text { tado ) } 12 \text { y tras } 3 \text { meses } \\
\text { después de un programa } \\
\text { de ejercicio aeróbico de } \\
\text { intensidad moderada }{ }^{10,12}\end{array}$ \\
\hline
\end{tabular}


narios, excepto el TAPA y el PRISCUS-PAQ, estudiaron la validez de constructo, mientras que la validez de criterio se realizó para todos los cuestionarios excepto LSA, MAT-SF y PFE. Además, existieron discrepancias sobre los instrumentos usados para establecer una $\mathrm{u}$ otra validez, por ejemplo, los podómetros fueron usados para establecer ambos tipos de validez. En relación a los instrumentos empleados para establecer la validez de criterio solo en uno de los cuestionarios que medían movilidad fue realizada esta medición mediante el DLW, mientras que tanto en los que medían actividad física como en los que medían gasto energético fueron empleados diversos métodos para establecer dicha validez como detectores de movimiento, DLW, $\mathrm{VO}_{2}$ max y los cuestionarios YPAS, PASE y CHAMPS. El estándar de oro (DLW) fue usado para validar el QAPSE, Zutphen y Minesotta Leisure Time. Sin embargo, existieron discrepancias entre la validez de criterio de algunos cuestionarios (YPAS, PASE y el Modified Baecke) con el DLW algunos artículos estipularon que no existía correlación ${ }^{12}$ pero otros decían que dicha correlación es moderada ${ }^{16,33}$.

La validez de constructo de los cuestionarios que midieron movilidad y actividad física se realizó principalmente con pruebas del desempeño EPESE (especialmente el SPPB en los cuestionarios que medían movilidad) y con detectores de movimiento. En cambio en los cuestionarios que medían gasto energético se empleó principalmente la ingesta calórica y el gasto energético diario o semanal para establecer dicha validez.

Solamente el CHAMPS, PASE e YPAS realizaron estudio de sensibilidad. El cuestionario CHAMPS demostró tener una buena sensibilidad al cambio después de 6 y 12 meses de programas de promoción de la AF y de resistencia y fortalecimiento en personas mayores. El YPAS demostró sensibilidad al cambio en estudios de 12 semanas y de 3 meses de duración $^{10,12}$. Y el PASE presentó sensibilidad al cambio en un estudio piloto de 6 semanas de duración ${ }^{10,12}$.

\section{DISCUSIÓN}

A través del análisis de los artículos seleccionados se pone de manifiesto que el método del agua doblemente marcada (DLW) es el más fiable y preciso para estudiar la movilidad, a través de la determinación de la energía gastada en la AF realizada, por ello se utiliza como estándar de oro para la validación de otros instrumentos ${ }^{12,16,18,25,26}$.

Entre los detectores de movimiento, podómetros y acelerómetros, son los acelerómetros el método más preciso para cuantificar la movilidad en las personas mayores a través de la energía gastada en los movimientos realizados ${ }^{16,19-21}$. Esta afirmación es corroborada por Gardner et al tras obtener una alta correlación con el DLW ${ }^{16}$. Sin embargo, los podómetros son más fáciles de usar y de colocar y, por lo tanto, presentan menos dificultades a la hora de seguir el protocolo de uso por parte de las personas mayores. Ambos instrumentos tienen dos problemas en común para captar la movilidad en las personas mayores, el primero es la dificultad que presentan para captar movimientos lentos o actividades de baja intensidad y el segundo es la falta de consenso existente sobre la localización idónea de ambos instrumentos, el lugar de colocación de ambos es la cintura ${ }^{16}$ pero dificulta la captación de los movimientos en las extremidades superiores. Para tratar de solventar ambos problemas varios autores proponen el uso de localizaciones alternativas como el tobillo en el caso de los podómetros ${ }^{48} \mathrm{o}$ el uso en muñeca o tobillo en los acelerómetros combinado con un acelerómetro en cintura ${ }^{21}$.

Existen acelerómetros o podómetros más adecuados para medir la movilidad en las personas mayores que otros. Así el The Step Watch es un podómetro más efectivo para medir la movilidad en las personas mayores que el más usado en la mayoría de los estudios que es el Yamax ${ }^{19,48}$, y entre los acelerómetros los más usados son el Catrac, Tritac y $\mathrm{CSA}^{16,21}$. Sin embargo el Caltrac es un acelerómetro poco apropiado para determinar la energía gastada en individuos mayores, ya que la 
subestima en un 50-55\% respecto del método de agua doblemente marcada ${ }^{16}$. En cuanto a su uso, los podómetros son los detectores de movimiento más empleados en las personas mayores debido a su fácil uso y al precio elevado y a la mayor complejidad de uso de los acelerómetros ${ }^{21}$. Esta afirmación se sustenta en dos revisiones sobre acelerómetros y podómetros encontrados, en la que 28 artículos miden la movilidad de las personas mayores con podómetro ${ }^{19}$ y solo 10 lo hacen con acelerómetros ${ }^{22}$. Además, los estudios consultados muestran que los tamaños muestrales empleados en los estudios con acelerómetros son muy reducidos, limitando la validez externa de dichos instrumentos ${ }^{22,23}$. En cambio su uso está más extendido para la validación de cuestionarios de movilidad.

Las medidas objetivas de la movilidad aportan datos objetivos más precisos y fiables para la medición de la movilidad de las personas mayores que los cuestionarios, además detectan in situ o precozmente alteraciones en la movilidad, permitiendo amortiguar el impacto de dichas limitaciones en la salud. Estos instrumentos de medición se desarrollan como respuesta a la falta de precisión de las medidas autocumplimentadas o cuestiona$\operatorname{rios}^{28}$, además precisan de muy poco tiempo para su realización y tienen un lenguaje limitado y sencillo que permite su adaptación a diversos entornos culturales ${ }^{17,28}$. Las limitaciones de estos instrumentos se refieren más a la necesidad de un equipamiento específico, un espacio adecuado para la realización de las pruebas y la imposibilidad de utilizarlos en personas con discapacidades severas o con determinadas enfermedades que a los problemas económicos que ocasiona su utilización. Sin embargo, son pocos los estudios que usan este instrumento de manera individual para medir la movilidad en las personas mayores, lo que se debe a que el mayor número de autores entiende que actividades como caminar suponen un mayor constructo de la movilidad que el desarrollo de una batería de actividades ${ }^{17}$. A pesar de estos datos, las pruebas objetivas como el SPPB o 400 meters test son de las más empleadas y recomendadas por los investigadores ${ }^{29,31,50}$, además de utilizarse en varios estudios epidemiológicos en personas mayores, estudios EPESE y el Health ABC respectivamente ${ }^{28}$, y en el caso del SPPB alcanzando los estándares de calidad recomendados dos de los tres tests y solo moderado el test del equilibrio ${ }^{49}$. Por lo tanto, estos instrumentos son unas herramientas adecuadas para medir la movilidad en personas mayores por la objetividad y rapidez de sus resultados, la facilidad de adaptación a distintos entornos culturales, la escasa duración de la prueba y la posibilidad de realizarla en muestras de población grandes debido al bajo coste económico. Todo ello compensa el mayor equipamiento y entrenamiento necesarios para su realización.

Los cuestionarios son los instrumentos más utilizados en la medición de la movilidad y de la AF, debido a su facilidad de uso y de administración, a que no requieren de un equipamiento especial y a su buena relación costebeneficio ${ }^{16}$. Además, la información que se obtiene con ellos, aunque indirecta, es de gran relevancia y utilidad ${ }^{16}$. Sin embargo, aportan datos subjetivos cuya precisión es menor que la de otros métodos debido a que están centrados en percepciones subjetivas de los individuos, están influenciados por problemas cognitivos y de memoria, su comprensión depende del nivel educativo y porque las personas encuestadas tienden a responder lo socialmente correcto ${ }^{16,17}$. Debido a estos problemas, algunos autores como Garatachea $\mathrm{N}$ et $a l^{16}$ aseguran que los peores niveles de movilidad de las personas mayores pueden deberse a mediciones poco precisas de los cuestionarios en este grupo de edad. Es cierto que esos problemas influyen en la realización de los cuestionarios por parte de las personas mayores, pero la presencia de un entrevistador entrenado debería mitigar parcialmente este efecto. Además, los malos resultados obtenidos reflejan el efecto que tienen las variables cognitivas y educativas en la movilidad de las personas mayores y no la mayor o menor precisión de los instrumentos de medida de la movilidad. 
La comparación de todos los instrumentos de medida de movilidad identificados y de sus ventajas y limitaciones muestra que:

- Respecto a la utilización, los cuestionarios, podómetros y pruebas objetivas de la movilidad son los instrumentos más empleados para medir la movilidad en las personas mayores. Así, en la revisión de de Jane Chung et $\mathrm{al}^{17}$ se encuentra que de los 103 artículos consultados, 68 son para cuestionarios y 17 para las pruebas objetivas. En relación a los podómetros, hallamos un total de 28 artículos que estudian la movilidad en 4.441 personas mayores ${ }^{19}$. En cambio, el número de artículos que usan el DLW y los acelerómetros son $218^{26}$ y $4^{11,21-23}$ respectivamente. Además, aunque los cuestionarios y los podómetros son instrumentos menos precisos que las pruebas objetivas y los acelerómetros, son las más usadas por su sencillez, precio y fácil uso por las personas mayores. Pruebas más precisas como el acelerómetro carecen de una adecuada adaptación a esta población.

- La elección de los instrumentos más idóneos en los estudios revisados se basa en el objetivo a alcanzar, así como en las características de la población estudiada ${ }^{17,28}$. Las alteraciones cognitivas pueden influir en los resultados, el efecto de estos problemas se amortiguaría con la presencia de una persona entrenada para realizar la entrevista. En cuanto al objetivo, las investigaciones buscan obtener información que englobe todos los aspectos de las personas, por ello es importante obtener tanto datos subjetivos basados en las percepciones como datos objetivos mediante los acelerómetros o por las medidas objetivas de la movilidad.

Una de las limitaciones de este trabajo es que no se realizó un análisis de la calidad de los artículos incluidos en la revisión y que en la extracción de datos, aunque fue consensuada por los autores, no se realizó un análisis por pares.

A partir del análisis expuesto, proponemos para la medida de la movilidad en las perso- nas mayores el uso mixto de instrumentos objetivos y subjetivos, de hecho, varios estudios emplean el uso combinado de ambos métodos para medir la movilidad en las personas mayores ${ }^{17}$.

Ésta propuesta se realiza tras analizar que los acelerómetros son poco usados en las personas mayores y que la información aportada por los podómetros es escasa y carente de relevancia. En cambio, las medidas objetivas nos aportan datos objetivos casi instantáneos de la movilidad de las personas mayores, que permiten detectar precozmente niveles de limitación de movilidad mientras que la elección de los cuestionarios se debe a su facilidad de uso, a su bajo coste y a los valiosos datos aportados sobre movilidad y factores sociodemográficos. Estos últimos son esenciales para comprender los múltiples factores que afectan a la movilidad de las personas mayores. Además, ambas pruebas las puede realizar un entrevistador entrenado, lo que amortigua el efecto de los problemas cognitivos y educativos en los resultados de movilidad.

Los instrumentos de medición seleccionados para las medidas objetivas es el SPPB debido a que es un test más completo y posee mejores características psicométricas y respecto a los cuestionarios, la versión corta del Minnesota Leisure Time Physical Activity es la que posee mayor fiabilidad test-rest, además de tener la mejor validez de criterio de los tres cuestionarios validados en población española.

\section{BIBLIOGRAFÍA}

1. Satariano WA, Guralnik JM, Jackson RJ, Marottoli RA, Phelan EA, Prohaska TR. Mobility and aging: new directions for public health action. Am J Public Health. 2012;102(8):1508-1515.

2. Webber SC, Porter MM, Menec VH. Mobility in older adults: a comprehensive framework. Gerontologist 2010;50(4):443-450.

3. Seidel D, Brayne C, Jagger C. Limitations in physical functioning among older people as a predictor of subsequent disability in instrumental activities of daily living. Age Ageing. 2011; 40 (4): 463-469. 
4. Hurtig-Wennlof A, Hagstromer M, Olsson LA. The International Physical Activity Questionnaire modified for the elderly: aspects of validity and feasibility. Public Health Nutr. 2010;13(11):1847-1854.

5. Bauman A, Bull F, Chey T, Craig CL, Ainsworth BE, Sallis JF, et al. The International Prevalence Study on Physical Activity: results from 20 countries. Int J Behav Nutr Phys Act. 2009; 6:21.

6. Arroyo P, Lera, Sánchez H, Bunout D, Santos J, Albala C. Indicadores antropométricos, composición corporal y limitaciones funcionales en ancianos. Rev MédiChile. 2007; 135(7): 846-854.

7. Miszkurka M, Zunzunegui MV, Langlois EV, Freeman EE, Kouanda S, Haddad S. Gender differences in mobility disability during young, middle and older age in West African adults. Glob Public Health. 2012;7(5):495-508.

8. Craig CL, Marshall AL, Sjostrom M, Bauman AE, Booth ML, Ainsworth BE, et al. International physical activity questionnaire: 12 -country reliability and validity. Med Sci Sports Exerc. 2003;35(8):1381-1395.

9. Baker PS, Bodner EV, Allman RM. Measuring lifespace mobility in community-dwelling older adults. J Am Geriatr Soc. 2003;51(11):1610-1614.

10. Harada ND, Chiu V, King AC, Stewart AL. An evaluation of three self-report physical activity instruments for older adults. Med Sci Sports Exerc. 2001;33(6):962970.

11. Troiano RP, Berrigan D, Dodd KW, Masse LC, Tilert T, McDowell M. Physical activity in the United States measured by accelerometer. Med Sci Sports Exerc .2008;40(1):181-188.

12. Guirao-Goris JA, Cabrero-García J, Moreno Pina JP, Muñoz-Mendoza CL. Revisión estructurada de los cuestionarios y escalas que miden la actividad física en los adultos mayores y ancianos. Gac Sanit . 2009; 23(4): 334.e51-334.e67.

13. Mantilla Toloza SC, Gómez Conesa A. El cuestionario internacional de actividad física. Un instrumento adecuado en el seguimiento de la actividad física poblacional. Rev Iberoam Fisioter Kinesol. 2007;10(1):48-52.

14. Ruiz Comellas A, Guillem P, Baena Díez JM, Mundet Tudurí X, Alzamora Sas T, Elosua R, et al . Validación de una versión reducida en español del cuestionario de actividad física en el tiempo libre de Minnesota (VREM). Rev Esp Salud Publica. 2012;86(5): 495-508.

15. Higgins JPT, Green S (editors). Manual Cochrane de revisiones sistemáticas de intervenciones. Version 5.1.0 [updated March 2011]. The Cochrane Collaboration, 2011. Disponible en: www.cochrane-handbook.org.
16. Garatachea N, De Paz-Fernández JA. Cuantificación de la actividad física en personas mayores. Rev Esp Geriatr Gerontol. 2005;49:47-52.

17. Chung J, Demiris G, Thompson HJ. Instruments to Assess Mobility Limitation in Community-Dwelling Older Adults: A Systematic Review. J Aging Phys Act. 2014; 23(2): 298-313

18. Park J, Kazuko IT, Kim E, Kim J, Yoon J. Estimating free-living human energy expenditure: Practical aspects of the doubly labeled water method and its applications. Nutr Res Pract. 2014;8(3):241-8.

19. Tudor-Locke C, Hart TL, Washington TL: Expected values for pedometer-determined physical activity in older populations populations. Int J Behav Nutr Phy. 2009, 6:59.

20. Tudor-Locke C, Williams JE, Reis JP, Pluto D. Utility of pedometers for assessing physical activity: construct validity. Sports Med. 2004;34(5):281-91.

21. Garatachea N, Torres Luque G, Gonzalez Gallego J. Physical activity and energy expenditure measurements using accelerometers in older adults. Nutr Hosp. 2010;25(2):224-230.

22. Cheung VH, Gray L, Karunanithi M. Review of accelerometry for determining daily activity among elderly patients. Arch Phys Med Rehabil. 2011;92(6):998-1014.

23. Plasqui G, Westerterp KR. Physical activity assessment with accelerometers: an evaluation against doubly labeled water. Obesity (Silver Spring). 2007; 15(10):2371-9.

24. Aoyagi Y, Shephard RJ. Sex differences in relationships between habitual physical activity and health in the elderly: practical implications for epidemiologists based on pedometer/accelerometer data from the Nakanojo Study. Arch Gerontol Geriatr. 2013;56(2):327-38.

25. Hertogh EM, Monninkhof EM, Schouten EG, Peeters PH, Schuit AJ. Validity of the Modified Baecke Questionnaire: comparison with energy expenditure according to the doubly labeled water method. Int J Behav Nutr Phys Act. 2008;5:30.

26. Yamada Y, Noriyasu R, Yokoyama K, Osaki T, Adachi T, Itoi A, et all. Association between lifestyle and physical activity level in the elderly: a study using doubly labeled water and simplified physical activity record. Eur J Appl Physiol. 2013;113(10):2461-71.

27. Harris TJ, Owen CG, Victor CR, Adams R, Ekelund $\mathrm{U}$, Cook DG. A comparison of questionnaire, accelerometer, and pedometer: measures in older people. Med Sci Sports Exerc. $2009 ; 41(7): 1392-402$. 
28. Savino E, Volpato S, Zuliani G, Guralnik JM. Assessment of mobility status and risk of mobility disability in older persons. Curr Pharm Des. 2014;20(19):3099113.

29. Panzer VP, Wakefield DB, Hall CB, Wolfson LI. Mobility assessment: sensitivity and specificity of measurement sets in older adults. Arch Phys Med Rehabil. 2011;92(6):905-12.

30. Butler AA, Menant JC, Tiedemann AC, Lord SR. Age and gender differences in seven tests of functional mobility. J Neuroeng Rehabil. $2009 ; 6: 31$.

31. Rejeski WJ, Marsh AP, Anton S, Chen SH, Church T, Gill TM, et all. The MAT-sf: clinical relevance and validity.J Gerontol A Biol Sci Med Sci. 2013;68(12):1567-74.

32. Rejeski WJ, Ip EH, Marsh AP, Barnard RT. Development and validation of a video-animated tool for assessing mobility. J Gerontol A Biol Sci Med Sci. 2010;65(6):664-671.

33. Voorrips LE, Ravelli AC, Dongelmans PC, Deurenberg P, Van Staveren WA. A physical activity questionnaire for the elderly. Med Sci Sports Exerc. 1991;23(8):974979.

34. Stel VS, Smit JH, Pluijm SM, Visser M, Deeg DJ, Lips P. Comparison of the LASA Physical Activity Questionnaire with a 7-day diary and pedometer. J Clin Epidemiol. 2004;57(3):252-258.

35. Peel C, Sawyer Baker P, Roth DL, Brown CJ, Brodner EV, Allman RM. Assessing mobility in older adults: the UAB Study of Aging Life-Space Assessment. Phys Ther. 2005;85(10):1008-1119.

36. Bonnefoy M, Kostka T, Berthouze SE, Lacour JR. Comparative study of daily energy expenditure measured by physical activity questionnaire (QAPSE) and physical fitness (VO 2max) in the elderly. Reprod Nutr Dev. 1996;36(4): 446-447.

37. De Abajo S, Larriba R, Marquez S. Validity and reliability of the Yale Physical Activity Survey in Spanish elderly. J Sports Med Phys Fitness. 2001;41(4):479-85.

38. Guerra RO, Oliveira BS, Alvarado BE, Curcio CL, Rejeski WJ, Marsh AP, et all. Validity and applicability of a video-based animated tool to assess mobility in elderly Latin American populations. Geriatr Gerontol Int. 2014: 864-863.

39. Devereaux Melillo K, Williamson E, Futrell M, Chamberlain C. A self-assessment tool to measure older adults' perceptions regarding physical fitness and exercise activity. J Adv Nurs. 1997;25(6):1220-6.
40. Moschny A, Platen P, Klaassen-Mielke R, Trampisch $\mathrm{U}$, Hinrichs T. Physical activity patterns in older men and women in Germany: a cross-sectional study. BMC Public Health. 2011;11:559.

41. Trampisch US, Platen P, Moschny A, Wilm S, Thiem U, Hinrichs T. Measurement of physical activity in older adults. Correlation between the PRISCUS-PAQ and accelerometry. Z Gerontol Geriatr. 2012;45(3):212-7.

42. Trampisch U, Platen P, Burghaus I, Moschny A, Wilm $\mathrm{S}$, Thiem U, et all. Reliability of the PRISCUS-PAQ. Questionnaire to assess physical activity of persons aged 70 years and older. Z Gerontol Geriatr. 2010;43(6):399406.

43. Topolski TD, LoGerfo J, Patrick DL, Williams B, Walwick J, Patrick MB. The Rapid Assessment of Physical Activity (RAPA) among older adults. Prev Chronic Dis. 2006;3(4):A118.

44. Mayer CJ, Steinman L, Williams B, Topolski TD, LoGerfo J. Developing a Telephone Assessment of Physical Activity (TAPA) questionnaire for older adults. Prev Chronic Dis. 2008 Jan;5(1):A24.

45. Bijnen FC, Feskens EJ, Caspersen CJ, Nagelkerke N, Mosterd WL, Kromhout D. Baseline and previous physical activity in relation to mortality in elderly men: the Zutphen Elderly Study. Am J Epidemiol. 1999;150(12):1289-1296.

46. Westerterp KR, Saris WH, Bloemberg BP, et al. Validation of the Zutphen Physical Activity Questionnaire for the Elderly with doubly labeled water. (Abstract). Med Sci Sports Exerc. 1992;24(suppl):S68.

47. Vega-Lopez S, Chavez A, Farr KJ, Ainsworth BE. Validity and reliability of two brief physical activity questionnaires among Spanish-speaking individuals of Mexican descent. BMC Research Notes. 2014; 7:29.

48. Bergman RJ, Bassett DR Jr, Muthukrishnan S, Klein DA. Validity of 2 devices for measuring steps taken by older adults in assisted-living facilities. J Phys Act Health. 2008;5 Suppl 1:S166-75.

49. Cabrero García J, Reig A, Muñoz CL, Cabañero MJ, Ramos JD, Richard M, et al. Reproducibilidad de la batería EPESE de desempeño físico en Atención Primaria. Anal Modifcación conducta 2007, 33: 67-83.

50. Ávila-Funes JA, Gray-Donald K, Payette H. Medición de las capacidades físicas de adultos mayores de Quebec: un análisis secundario del estudio NuAge. Salud Pública Méx. 2006; 48( 6 ): 446-454. 